\title{
Reconfigurable MRI technology for low-SAR imaging of deep brain stimulation at 3T: Application in bilateral leads, fully-implanted systems, and surgically modified lead trajectories
}

\author{
Ehsan Kazemivalipour ${ }^{\mathrm{a}, \mathrm{b}, \mathrm{c}}$, Boris Keil ${ }^{\mathrm{d}}$, Alireza Vali ${ }^{\mathrm{a}}$, Sunder Rajan ${ }^{\mathrm{e}}$, Behzad Elahi ${ }^{\mathrm{f}}$, \\ Ergin Atalar ${ }^{\mathrm{b}, \mathrm{c}}$, Lawrence L. Wald ${ }^{g}$, Joshua Rosenow ${ }^{\mathrm{h}}$, Julie Pilitsis ${ }^{\mathrm{i}}$, Laleh Golestanirad ${ }^{\mathrm{a}, \mathrm{j},}$ \\ ${ }^{a}$ Department of Radiology, Feinberg School of Medicine, Northwestern University, Chicago, IL, USA \\ ${ }^{\mathrm{b}}$ Department of Electrical and Electronic Engineering, Bilkent University, Ankara, Turkey \\ ${ }^{\mathrm{c}}$ National Magnetic Resonance Research Center (UMRAM), Bilkent University, Ankara, Turkey \\ ${ }^{\mathrm{d}}$ Department of Life Science Engineering, Institute of Medical Physics and Radiation Protection, Giessen, Germany \\ ${ }^{\mathrm{e}}$ Division of Biomedical Physics, Office of Science and Engineering Laboratories, Center for Devices and Radiological Health, U.S. Food and Drug Administration, Silver \\ Spring, MD, USA \\ ${ }_{\mathrm{f}}^{\mathrm{f}}$ Department of Neurology, Bryan Health, Lincoln, NE, USA \\ ${ }^{g}$ A. A. Martinos Center for Biomedical Imaging, Massachusetts General Hospital, Boston, MA, USA \\ ${ }^{\mathrm{h}}$ Department of Neurosurgical Surgery, Feinberg School of Medicine, Northwestern University, Chicago, IL, USA \\ ${ }^{i}$ Department of Neurosurgery, Albany Medical Center, Albany, NY, USA \\ ${ }^{\mathrm{j}}$ Department of Biomedical Engineering, McCormick School of Engineering, Northwestern University, Evanston, IL, USA
}

\section{A R T I C L E I N F O}

\section{Keywords:}

Deep brain stimulation (DBS)

Individualized medicine

In-silico medicine

Finite element

Magnetic resonance imaging (MRI)

MRI safety

Neuromodulation

Neurostimulation

Medical implants

Reconfigurable MRI

Simulations

Specific absorption rate (SAR)

MRI coils

\begin{abstract}
A B S T R A C T
Patients with deep brain stimulation devices highly benefit from postoperative MRI exams, however MRI is not readily accessible to these patients due to safety risks associated with RF heating of the implants. Recently we introduced a patient-adjustable reconfigurable coil technology that substantially reduced local SAR at tips of single isolated DBS leads during MRI at $1.5 \mathrm{~T}$ in 9 realistic patient models. This contribution extends our work to higher fields by demonstrating the feasibility of scaling the technology to $3 \mathrm{~T}$ and assessing its performance in patients with bilateral leads as well as fully implanted systems. We developed patient-derived models of bilateral DBS leads and fully implanted DBS systems from postoperative CT images of 13 patients and performed finite element simulations to calculate SAR amplification at electrode contacts during MRI with a reconfigurable rotating coil at 3T. Compared to a conventional quadrature body coil, the reconfigurable coil system reduced the SAR on average by $83 \%$ for unilateral leads and by $59 \%$ for bilateral leads. A simple surgical modification in trajectory of implanted leads was demonstrated to increase the SAR reduction efficiency of the rotating coil to $>90 \%$ in a patient with a fully implanted bilateral DBS system. Thermal analysis of temperature-rise around electrode contacts during typical brain exams showed a 15-fold heating reduction using the rotating coil, generating $<1^{\circ} \mathrm{C}$ temperature rise during $\sim 4$-min imaging with high-SAR sequences where a conventional CP coil generated $>10^{\circ} \mathrm{C}$ temperature rise in the tissue for the same flip angle.
\end{abstract}

\section{Introduction}

Deep brain stimulation (DBS) is an FDA-approved neurostimulation procedure treating drug resistant Parkinson's disease (PD), essential tremor (ET), and dystonia (Flora et al., 2010; Hubble et al., 1996; Blomstedt et al., 2007; Kumar et al., 1999; Ostrem and Starr, 2008), with applications rapidly expanding to neuropathic pain (Owen et al., 2007; Boccard et al., 2012), obsessive compulsive disorder (Gabriels et al.,
2003; Greenberget al, 2008), epilepsy (Boonet al, 2007), and Alzheimer's disease (Laxton and Lozano, 2013). Although decades have passed since the inception of DBS and its clinical applications have grown exponentially, its biophysical mechanisms remain unclear. Uncertainties remain about which circuits are affected, which exact neural populations need to be targeted, and the most efficacious stimulation protocol (Lozanoet al, 2019). There is a consensus that meticulous use of neuroimaging, both for target verification and for postoperative monitoring of functional

\footnotetext{
* Corresponding author. Department of Radiology, Feinberg School of Medicine, Northwestern University, Chicago, IL, USA.

E-mail address: laleh.rad1@northwestern.edu (L. Golestanirad).
} 
changes induced in the affected brain networks can help interpreting clinical outcomes and design enhanced therapeutic protocols (Williams et al., 2014; Cui and Ling, 2016). Due to its excellent soft tissue contrast and non-invasive nature, magnetic resonance imaging (MRI) is excellently poised to answer open questions regarding DBS mechanism and targeting. Unfortunately, the interaction of radiofrequency (RF) fields of MRI scanners and implanted leads results in safety hazards that limit postoperative accessibility of MRI to these patients (Rezaiet al, 2005; Rezaiet al, 2004). Today, MRI of DBS patients is limited to MR-conditional devices which require pulse sequences with a whole-head SAR of $0.1 \mathrm{~W} / \mathrm{kg}$ (30 times below the FDA limit for imaging in the absence of conductive implants) and 3T systems are absolutely contraindicated (Medtronic, 2015; Jude Medical, 2018). This excludes sequences that are essential to rule out complications (e.g., diffusion and T2-weighted imaging to detect stroke and hemorrhage), state-of-the-art fast imaging techniques that have become an integral part of functional studies (e.g., simultaneous multi-slice imaging (Feinberg and Setsompop, 2013)), and novel high-precision electrode localization methods (e.g., zero-TE phase imaging (Ramani et al., 2018)).

The phenomenology of RF heating in the presence of conductive implants in the MRI environment has been studied extensively (Nitz et al., 2001; Park et al., 2007; Yeung et al., 2002; Buchli et al., 1988; Chou et al., 1997; Golestaniradet al, 2019a). The consensus is that the tangential component of the electric field $\left(E_{\tan }\right)$ along the length of elongated conductive implants acts as a local voltage source generating $\mathrm{RF}$ currents on the leads. When these currents are dissipated in the tissue at the exposed tip of the lead, local SAR amplification and temperature rise will occur which can damage the tissue. The past few years have witnessed a spike in feasibility studies investigating the applicability of parallel transmit methodologies to reduce the SAR during DBS MRI (McElcheran et al., 2015; McElcheran et al., 2017a; Eryaman et al., 2011; Eryamanet al, 2014; Wei et al., 2018; McElcheran et al., 2016; McElcheranet al, 2017; McElcheranet al, 2019), although such techniques have not been implemented in clinical settings yet. Recently, we introduced the concept of patient-adjustable reconfigurable MRI technology for DBS imaging at $1.5 \mathrm{~T}$. The system is based on a mechanically rotating transmitter which steers a zero-electric field onto the electrode, then images with a high SNR close-fitting receive array (Golestaniradet al, 2016; Golestaniradet al, 2017; Golestanirad et al., 2017). Numerical simulations with realistic models of single isolated DBS leads demonstrated virtual elimination of SAR amplification around electrode contacts in nine patient models (Golestaniradet al, 2017). With promising preliminary results at $1.5 \mathrm{~T}$, substantial interest has arisen toward scaling the technology to higher fields where imaging can render significant benefits. The present work sets the ground for the advancement of the reconfigurable MRI technology to 3T. We assess the scalability of the system for imaging at $127 \mathrm{MHz}$, evaluate its performance in patients with bilateral implants and fully implanted systems, and report for the first time, the result of modifications in the DBS surgical procedure that enhances the subsequent performance of the new coil. Finally, we discuss benefits of the technology in terms of possibility of using pulse sequences currently not accessible to DBS patients due to their high SAR.

Below is a brief description of motivations and novel contributions of this work.

\subsection{Is reconfigurable MRI technology scalable to $3 T$ ?}

Recent studies have demonstrated the feasibility of using a rotating transmit coil to reduce the SAR during MRI of DBS patients at 1.5T (Golestaniradet al, 2016; Golestaniradet al, 2017; Golestanirad et al., 2017). There is however, a strong incentive toward DBS imaging at 3T for target verification and ruling out complications. This is because $1.5 \mathrm{~T}$ MRI consistently underestimates the anterior and lateral boundaries of subthalamic nucleus and globus pallidus (DBS main target nuclei) compared to 3T fast gray matter $\mathrm{T} 1$ inversion recovery acquisitions (Kerl et al., 2012), and a clear delineation between healthy and diseased tissue that allows detection of intracranial hemorrhage is better achieved at 3T. As the reconfigurable coil works by steering a low E-field region of the transmit coil such that it maximally contains the implant trajectory, question arises as whether this low E-field region is "thick" enough at 3T to fully contain realistic leads trajectories. This is especially important considering that DBS leads have complex trajectories with substantially out-of-plane segments, and that the spatial homogeneity of MRI RF fields is reduced at higher frequencies.

\subsection{Applicability to bilateral leads}

To date, studies that have investigated shaping or steering of MRI electric fields to reduce the SAR during DBS imaging have focused on single (unilateral) implants (McElcheran et al., 2015; Eryamanet al, 2014; Wei et al., 2018; McElcheran et al., 2016; McElcheranet al, 2017; McElcheran et al., 2014; Eryamanet al, 2019). In practice however, most patients with Parkinson's disease undergo bilateral lead implantation (Ondo et al., 2001) and 52\% of patients with essential tremor who originally receive unilateral DBS eventually need bilateral stimulation (Tabaet al, 2010). In the context of reconfigurable MRI, the optimal coil rotation angle to minimize the SAR depends on individual lead trajectories (Golestaniradet al, 2017) meaning a rotating transmit coil has to be positioned at different angles to minimize the SAR of different leads. This raises the concern as whether the technique can ever be used in patients with bilateral implants. To our knowledge, the issue of RF heating of bilateral DBS leads has not been addressed in the context of E-field steering techniques, including techniques based on parallel transmit and dual-drive systems (McElcheran et al., 2015; Eryamanet al, 2014; Wei et al., 2018; McElcheran et al., 2016; McElcheranet al, 2017; McElcheran et al., 2014; Eryamanet al, 2019; McElcheran et al., 2017b).

\subsection{Isolated leads $v$ s fully implanted DBS systems}

DBS surgery is performed in two stages. First, electrodes are implanted at specific target nuclei (e.g., subthalamic nucleus and globus pallidus for PD, ventrointermedius nucleus for ET) and the other end of the lead is tucked under scalp for later connection to the neurostimulator (Fig. 1A). MRI is useful at this stage for electrode localization and target verification (Cui and Ling, 2016). In the next step, an implantable pulse

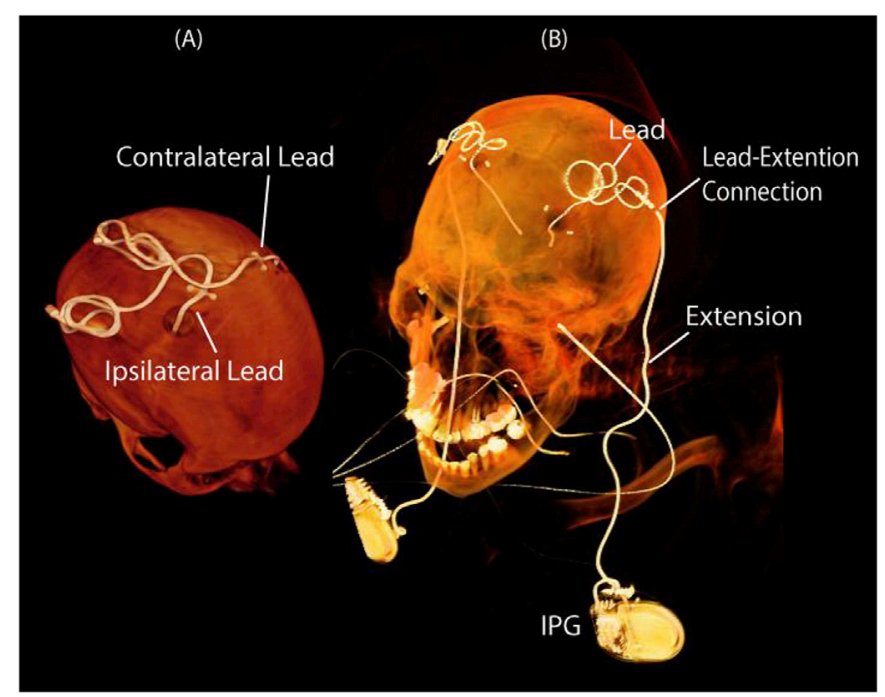

Fig. 1. CT images of patients with isolated and fully implanted DBS devices. A: Patient with bilateral leads prior their connection to the implantable pulse generator (IPG). Labels "contralateral" and "ipsilateral" are with respect to the body side that the IPG is planned to be implanted later. B: A patient with a fully implanted DBS system consisting of two IPGs, two leads, and two extension cables connecting leads to the IPGs. 
generator (IPG) is implanted in the chest or abdomen, and leads are connected to the IPG via subcutaneous extensions (Fig. 1B). Functional MRI studies that seek to disclose the neuromodulatory effect of DBS are performed on fully implanted systems (Hancuet al, 2018). To date, the performance of SAR reduction strategies based on E-field steering has been only investigated in isolated leads. It is established however that the presence, position, and configuration of the IPG has a significant effect on the RF heating (Matteiet al, 2008; Nordbecket al, 2009). Therefore, it is imperative to investigate the efficacy of field shaping techniques when applied on fully implanted DBS systems.

\subsection{Surgical lead management combined with rotating coil technology}

Unlike the meticulously planned intracranial trajectory of the electrode, for which almost every neurosurgeon follows textbook guidelines to determine the entry point on the skull and chose the angle of penetration to the target nuclei, there are no guidelines for the placement of extra cranial portions of the leads. Commercial DBS leads come in a series of fixed lengths at $30 \mathrm{~cm}, 40 \mathrm{~cm}$ and $50 \mathrm{~cm}$ which are longer than the distance from the surgical burr hole (where leads exists the skull) and the point where leads are connected to extensions (Fig. 1). Surgeons usually loop the leads arbitrarily on the skull to avoid positioning the excess material against soft tissue of the neck. This, causes a large variation in lead trajectories depending on surgeons' preference and practice style. The concept of surgical lead management to reduce the risk of MRI RF heating was first introduced by Baker in phantom studies (Baker et al., 2005), suggesting the use of external guides to incorporate concentric loops in the trajectory of DBS leads to reduce RF heating during MRI at $1.5 \mathrm{~T}$ and $3 \mathrm{~T}$. These findings were later corroborated in simulation studies (Golestanirad et al., 2016) and demonstrated to be practically implementable in clinic (Golestaniradet al, 2019a). Theoretically, lead management techniques can be modified for use in combination with E-field steering techniques to augment their benefit. This work reports the first result of implementing such surgical modifications tailored to enhance the performance of reconfigurable MRI technology.

In what follows we give a brief overview of the theory and concept of reconfigurable coil technology for low-SAR DBS imaging, discuss the details of coil design, numerical simulations, and patient models; evaluate the SAR reduction performance of the coil for unilateral and bilateral DBS leads as well as fully implanted systems; introduce a surgical lead management strategy that significantly enhances performance of the rotating coil; and discuss the sensitivity of coil positioning to operational errors and electrical properties of the tissue. Finally, we investigate the temperature rise in the tissue around tips of DBS leads during eight typical MRI exams in a patient will fully implanted DBS system scanned with the body coil as well as the rotating coil in its optimum position.

\section{An overview of patient-adjustable MRI coil technology}

An overview of the working principle of the reconfigurable MRI coil technology is given in Fig. 2, depicting a linearly-polarized (LP) rotating birdcage transmitter loaded with a human head implanted with a patientderived model of bilateral DBS leads. The electric field of the coil is given on a transverse plane passing through the center of the head. Such
(A)

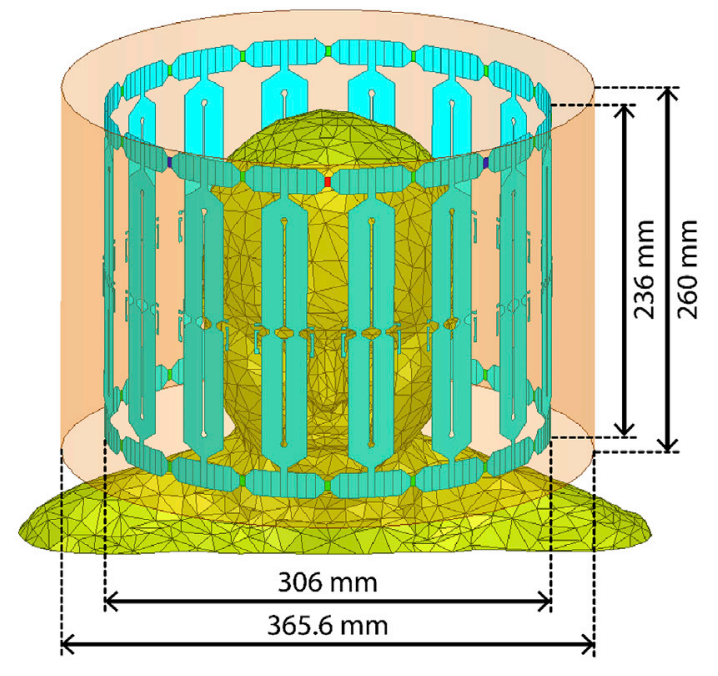

Fig. 2. Overview of the working principle of the rotating coil system. A linearly polarized birdcage transmitter has a slab-like region of low electric field. The orientation of this low E-field region can be steered by mechanically rotating the coil around patient's head. The heating of conductive wire implants can be minimized when they are maximally contained within this low field region. In this figure, the coil input power is adjusted to produce a mean $\mathrm{B}_{1}^{+}=2 \mu \mathrm{T}$ on a central axial plane passing through the head.

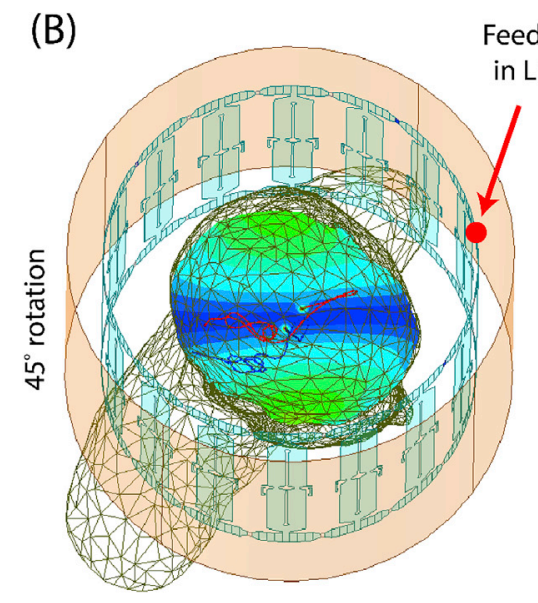

$1 \mathrm{~g}$-averaged $\mathrm{SAR}_{\max }=1.1 \mathrm{~W} / \mathrm{kg}$
Feeding port

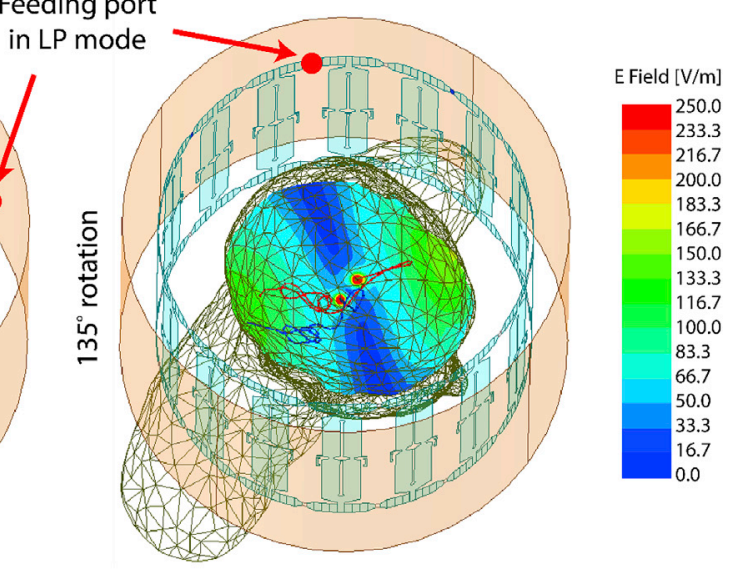

$1 \mathrm{~g}$-averaged $\mathrm{SAR}_{\max }=47.9 \mathrm{~W} / \mathrm{kg}$ 
linearly-polarized birdcage transmitter has a slab-like region of low electric field which can be steered to coincide with the implant trajectory by mechanically rotating the coil around patient's head. Fig. $2 \mathrm{~B}$ gives the maximum of 1g-averaged SAR around tips of left and right DBS leads as the coil rotates around the patient's head. As it can be observed, local SAR around DBS contacts can be minimized by positioning the coil at an optimum rotating angle such that the low E-field region maximally contains lead trajectories.

\section{Numerical simulations: rotating coil and patient models}

To assess the performance of the rotating coil system at 3T we constructed realistic lead models from postoperative computed tomography
(CT) images of 13 patients implanted with bilateral DBS leads. From these, 12 patients had isolated leads and one patient had a fully implanted DBS system with bilateral IPGs. The prospective use of imaging data for simulation and modeling was approved by the IRB at our institutions. Details of image segmentation and model construction were similar to our previous work (Golestaniradet al, 2019a). The coil was a shielded 16-rung high-pass linearly-polarized birdcage transmitter with a diameter of $306 \mathrm{~mm}$ and a length of $236 \mathrm{~mm}$, fed through a cable connected to the upper ring over a matching capacitor. The reference position for the feed $\left(\varphi=0^{\circ}\right)$ was set to be in front of patient's nose. The shield was an open solid copper cylinder with a length of $260 \mathrm{~mm}$ and a diameter of $365.6 \mathrm{~mm}$. ANSYS Electronics Desktop 18.2 (ANSYS Inc. Canonsburg PA) was used to implement numerical simulations. The coil
ID1

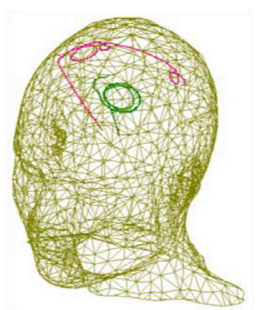

ID5

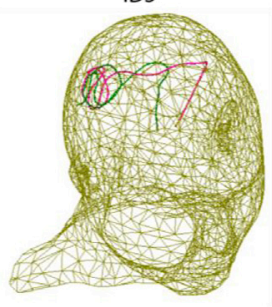

ID9

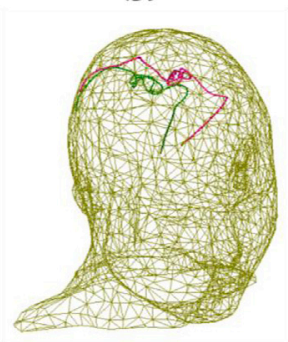

ID11

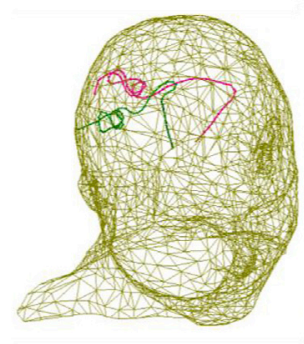

ID2

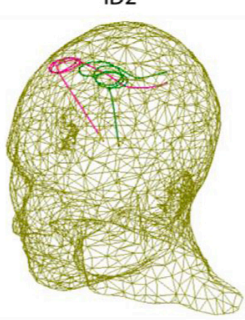

ID6

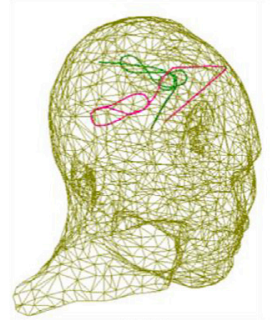

ID10

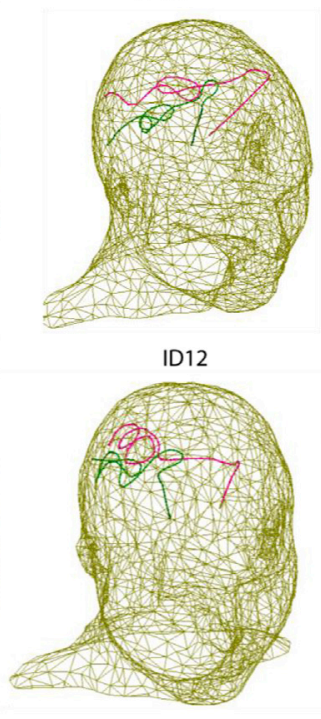

ID3

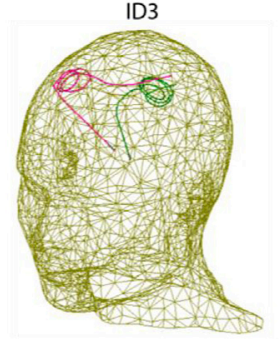

ID7

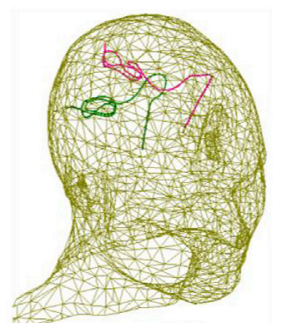

ID13

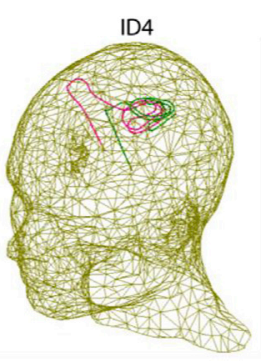

ID8

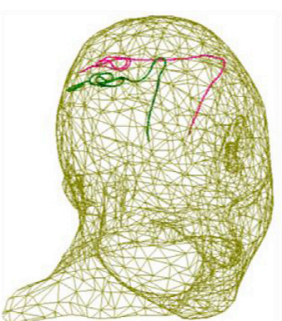

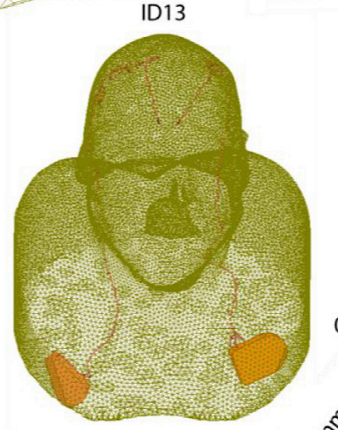

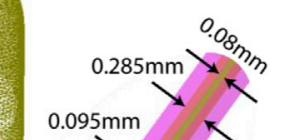

s.
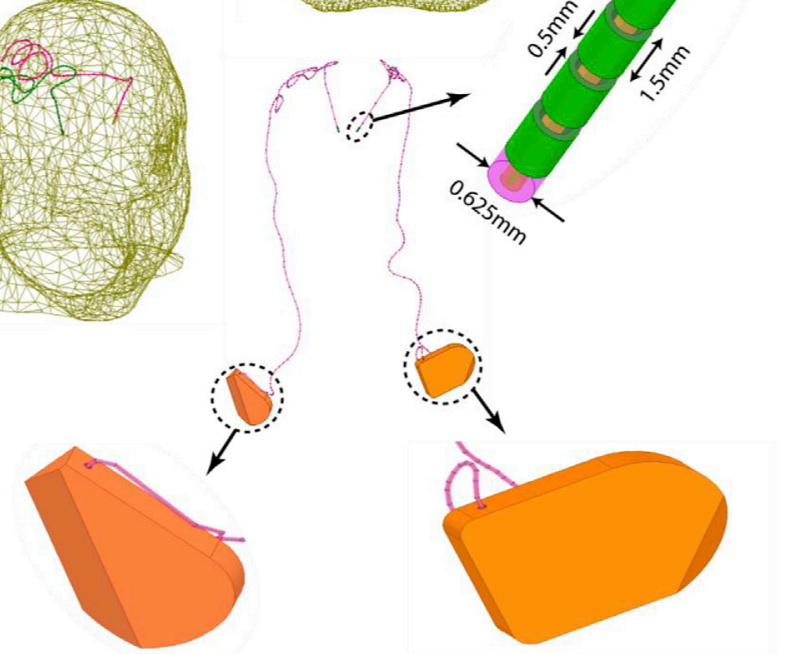

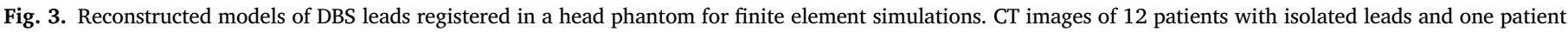
with a fully implanted system were used to extract lead trajectories. Models of electrode contacts and the insulation were built around each trajectory. 
was tuned and matched to operate at $127 \mathrm{MHz}$ using a combined finite element method and circuit analysis approach as described in previous works (Golestaniradet al, 2019a; Golestaniradet al, 2017; Golestanirad et al., 2017; Golestanirad et al., 2016; Kozlov and Turner, 2009; Golestaniradet al, 2019c).

Isolated lead models were registered to a homogeneous head phantom $\left(\varepsilon_{\mathrm{r}}=78, \sigma=0.47 \mathrm{~S} / \mathrm{m}\right)$ that extended to the base of neck. The fully implanted system was modeled using the silhouette of the patient constructed from head and chest CT images. Fig. 3 shows patient-derived models and details of DBS contact electrodes.

For each patient model, the coil was rotated around the head with $5.625^{\circ}$ increments and the maximum of 1 g-averaged SAR was calculated around tips of left and right DBS leads in a $20 \mathrm{~mm} \times 20 \mathrm{~mm} \times 20 \mathrm{~mm}$ cubic area that surrounded all four electrode contacts (Fig. 4). SAR values were calculated based on IEEE STD P1528.4 recommendation (IEEE, 2014) using the built-in SAR calculator module in ANSYS HFSS. Although there are FDA recommended limits of $3.2 \mathrm{~W} / \mathrm{kg}$ for the global head SAR, and $10 \mathrm{~W} / \mathrm{kg}$ for $10 \mathrm{~g}$-averaged local head SAR when operating in normal mode, there is no guideline for the local SAR in presence of medical implants. It is well established however, that implant-induced RF heating is a local phenomenon that occurs in the first few millimeters of the device and dies off fast (Matteiet al, 2007). For this reason, we calculated $1 \mathrm{~g}$-avereaged local SAR which gives a more focal description of heating in the vicinity of DBS leads.

A fine mesh resolution was enforced in the tissue surrounding electrode contacts with the mesh size $<0.7 \mathrm{~mm}$. The mesh size on metallic contacts and internal wires was set to be $<0.3 \mathrm{~mm}$. The computing space had $\sim 4$ million tetrahedral mesh after numerical convergence was achieved. Total computation time for each finite element simulation was $\sim 4 \mathrm{~h}$ on a Dell PowerEdge R740xd system with 1.5 TB RAM and two Intel(R) Xenon(R) Gold 6140 CPUs ( $2.30 \mathrm{GHz}, 18$ cores).

A total of 832 simulations were performed (13 patients $\times 64$ coil positions). To compare the SAR of linearly-polarized rotating coil with

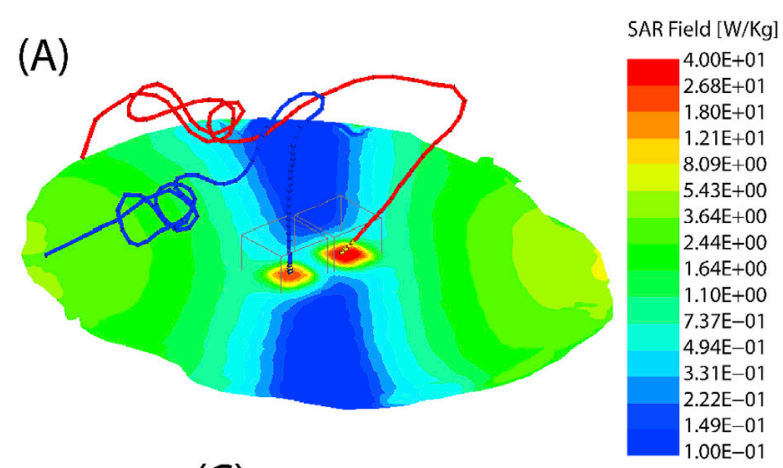

(C)

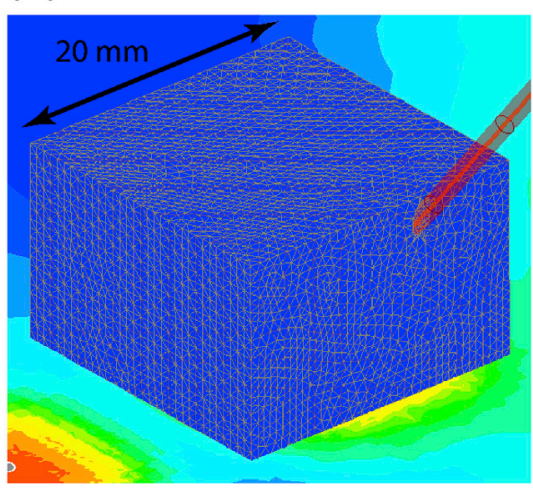

the SAR generated by a conventional circularly polarized (CP) body coil, we simulated a typical 3T high-pass birdcage coil with dimensions given in the literature (Yeo et al., 2011). To properly load the body coil, we registered isolated DBS leads into a homogeneous head and torso phantom that extended to the upper chest. Details of the body coil's geometry, head and torso phantom, and positioning of patient-derived DBS lead models in the body coil are given in the Supplement Fig. S1. For all simulations, the input power of the coils was adjusted to produce a $\mathrm{B}_{1}^{+}$on a central axial plane with a spatial mean of $2 \mu \mathrm{T}$.

\section{SAR reduction performance}

Fig. 5 (solid lines) gives the maximum of 1 -averaged SAR (Max1gSAR) around tips of right and left DBS electrodes for a full range of coil rotation angles. The Max1gSAR generated in the tissue by the body coil is also given for comparison (dashed-lines). For 11 patients, including the one with the fully-implanted system, we could find an optimum coil rotation angle that reduced the SAR at tips of both leads to a level below the Max1gSAR generated by the coil operated in CP mode.

\subsection{SAR reduction for unilateral leads}

To quantify the SAR-reduction performance of the coil we defined a metric called SAR-reduction efficiency (SRE) for each lead and at each coil rotation angle as:

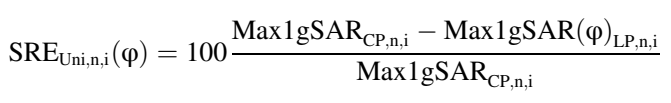

where Max1gSAR $\operatorname{CP}, \mathrm{n}, \mathrm{i}_{\text {is }}$ the maximum of $1 \mathrm{~g}$-averaged SAR at the tip of lead $\mathrm{i}$ ( $\mathrm{i}=$ right, left) in patient $\mathrm{n}$ generated by the $\mathrm{CP}$ body coil, and $\operatorname{Max} 1 \operatorname{gSAR}(\varphi)_{\mathrm{LP}, \mathrm{n}, \mathrm{i}}$ is the maximum of $1 \mathrm{~g}$-averaged SAR at the tip of lead $\mathrm{i}$ in patient $n$ generated by linearly-polarized rotating coil positioned at angle $\varphi$.

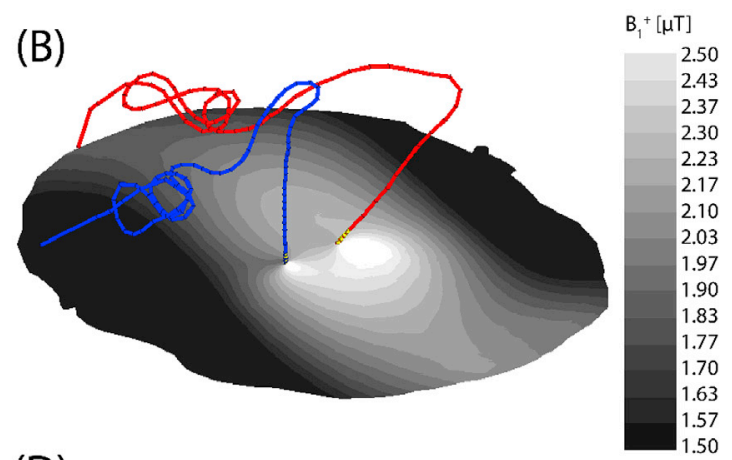

(D)

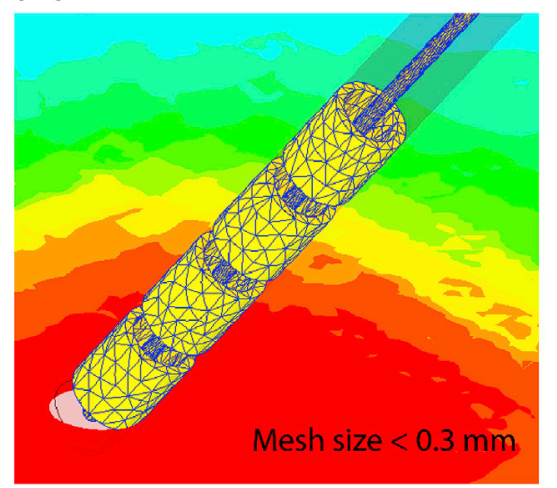

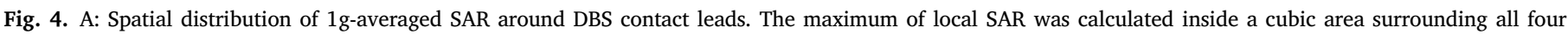

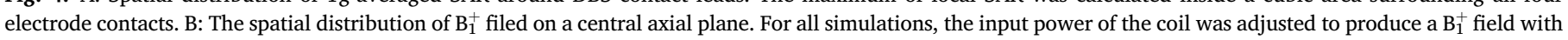

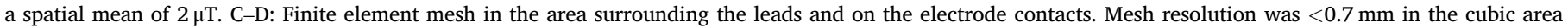
surrounding the leads and $<0.3 \mathrm{~mm}$ on the electrode contacts. 
IDI

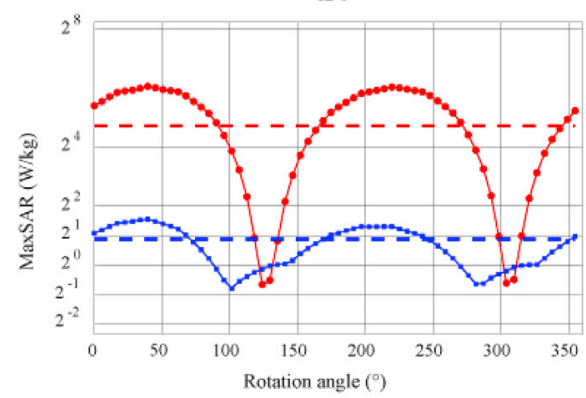

ID3

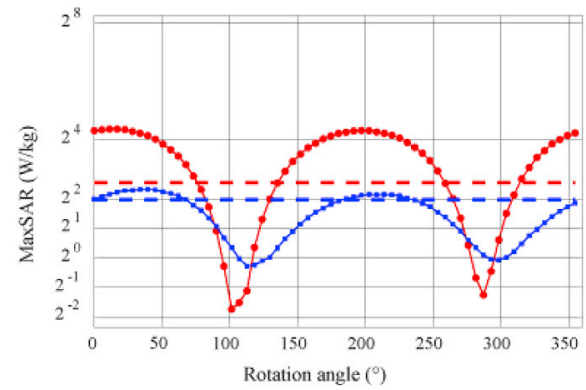

ID5

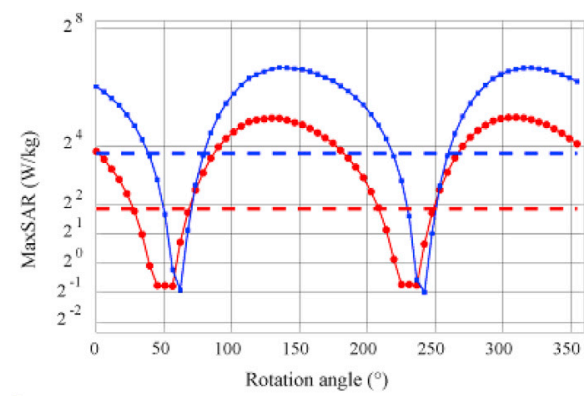

ID7

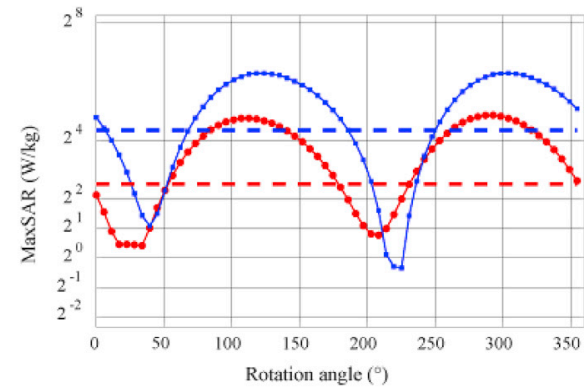

ID2

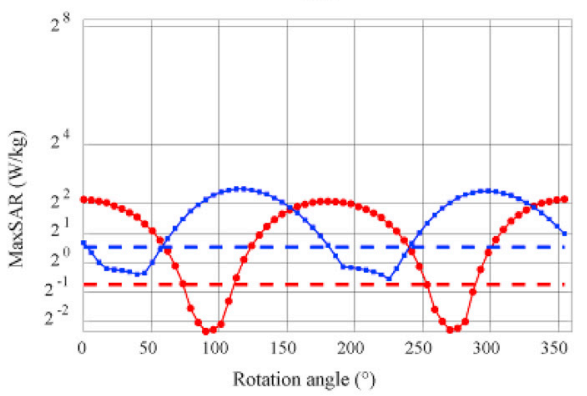

ID4

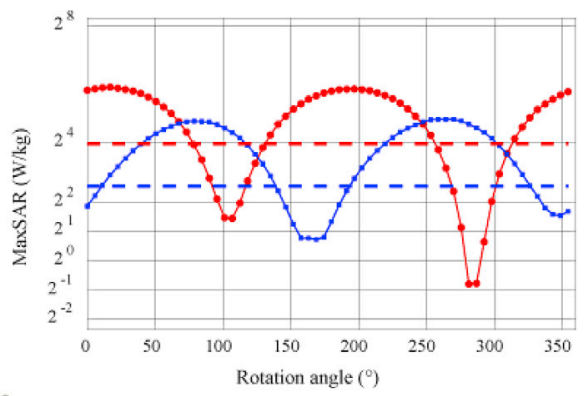

ID6

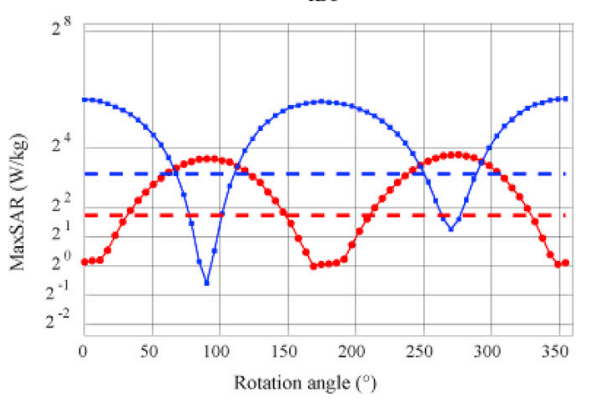

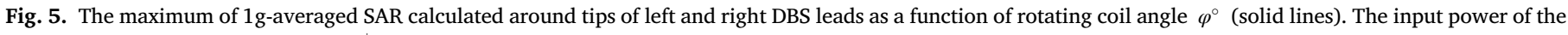

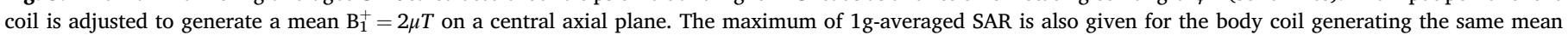
$\mathrm{B}_{1}^{+}=2 \mu \mathrm{T}$ (dashed-lines).

When considering each lead alone, an average SRE of $83.06 \% \pm 12.38 \%$ was achieved over all 26 leads. The unilateral SRE was positive for all leads, meaning that we could always find a rotating position that reduced the SAR at the tip of a unilateral lead to a value below the SAR generated by the body coil. As expected, the optimum coil rotation angle that minimized the SAR was different for each lead, emphasizing the importance of taking realistic trajectories into account (Greenberget al, 2008; Boonet al, 2007; Laxton and Lozano, 2013).

Fig. 5 also demonstrates the periodic nature of the SAR profile exhibiting two minima as the coil rotates a full circle. The mean value of the first optimum rotation angle was $73.99^{\circ} \pm 41.65^{\circ}$ averaged over all leads. From the practical point of view, this means that constructing the coil housing such that allows $180^{\circ}$ rotational maneuverability around a default feed position at $73.99^{\circ}$ will be sufficient to cover most typical DBS cases.

\subsection{SAR reduction for bilateral leads}

A bilateral SAR reduction metric can be defined as:

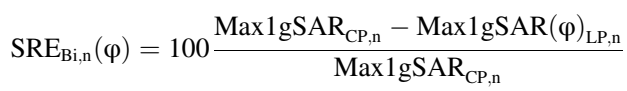

where the Max1gSAR ${ }_{\mathrm{CP} / \mathrm{LP}, \mathrm{n}}$ is the maximum of $1 \mathrm{~g}$-averaged SAR in the tissue of patient $\mathrm{n}$ at whichever lead that produces the higher SAR. When considering both leads, an average bilateral SRE of $59 \% \pm 38 \%$ was 
ID8

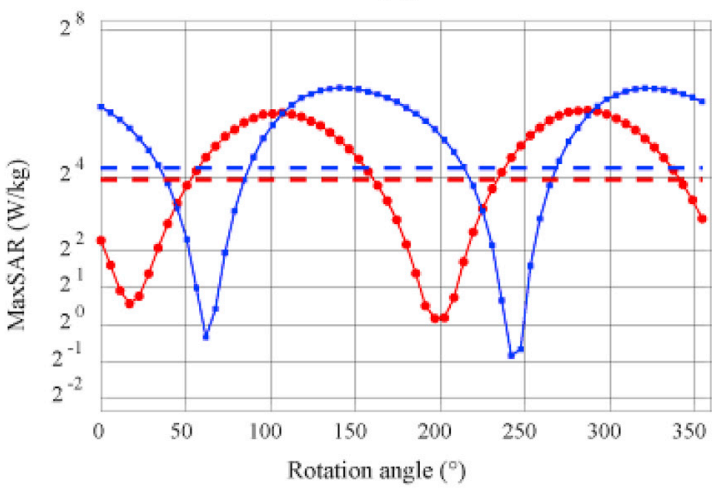

ID 10

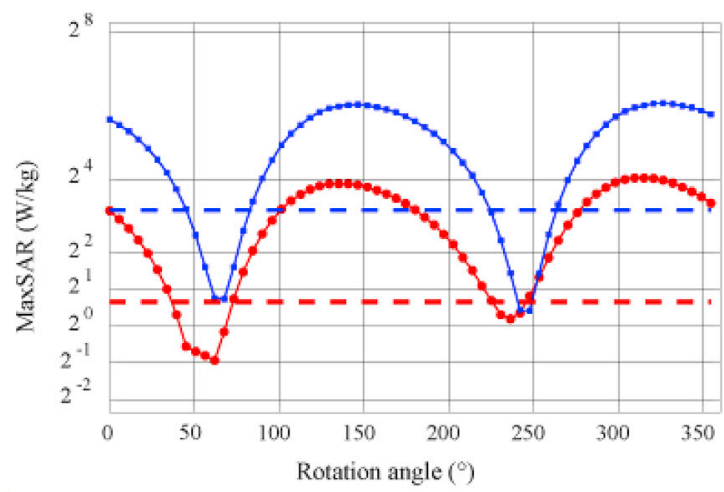

ID 12

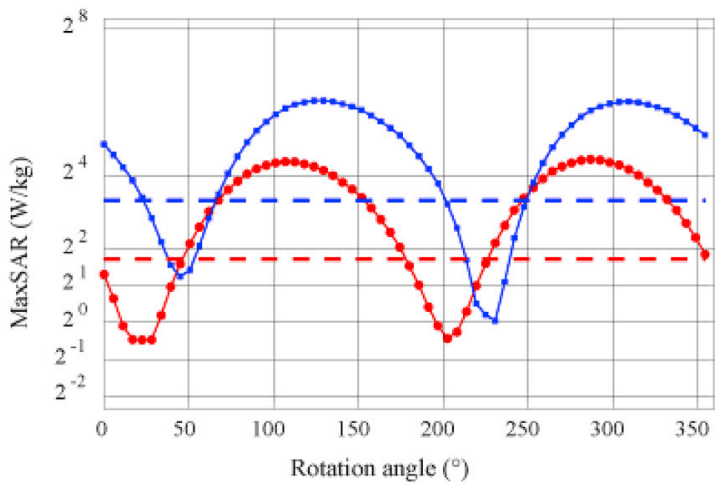

_- Linearly-polarized mode, Right-lead

__ Linearly-polarized mode, Left-lead
ID9

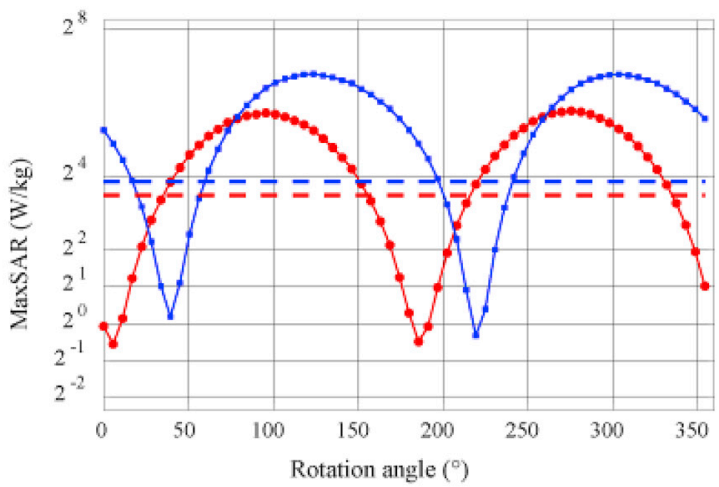

ID11

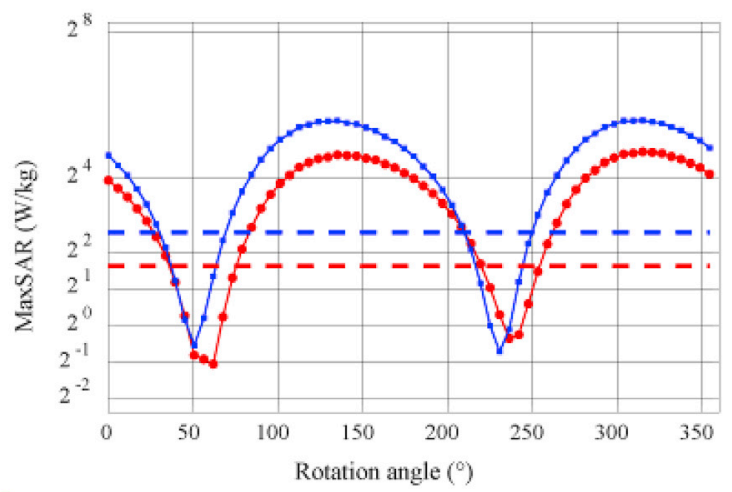

ID13

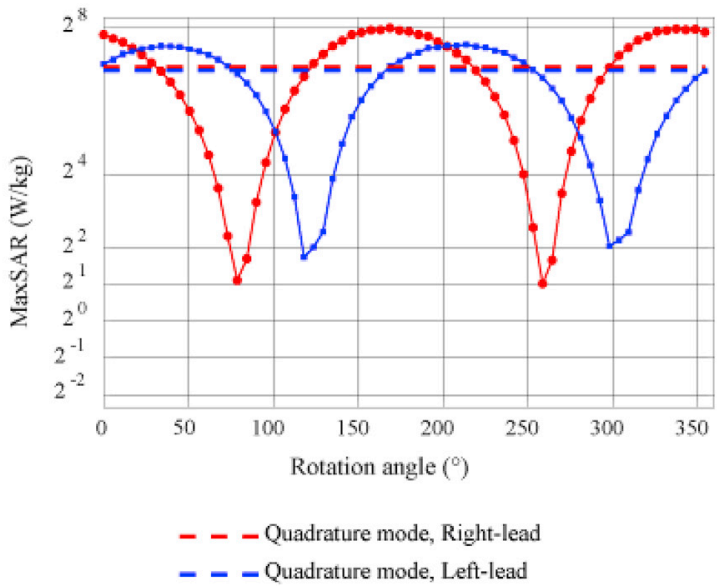

Fig. 5. (continued).

achieved over all patients. Table 1 gives the values of the maximum of $S R E_{U n i, n, i}$ and $S R E_{B i, n}$ for each patient and their corresponding optimum coil rotating angles.

\section{Surgical lead management to enhance the performance of reconfigurable coil}

The feasibility and effectiveness of surgeon-initiated improvements in the routing of DBS leads to mitigate the risk of MRI-induced RF heating has been recently brought into attention by our group (Golestaniradet al, 2019a). Regarding the reconfigurable coil technology, one can think of implementing similar surgical strategies that ensure optimal performance of the rotating coil for bilateral leads. This can be achieved by implementing a few modifications. First, incorporating concentric loops close to the surgical bur hole will further reduce the SAR amplification at tips of both left and right leads through cancelation of induced voltages as described in our previous work (Golestaniradet al, 2019a). Second, aligning and overlapping the trajectories of right and left leads and extension cables will allow the rotating coil to maximally contain both leads in its low E field region and thus minimizing the SAR at tips of both implants simultaneously. In cases where two IPGs are implanted bilaterally to stimulate left and right leads separately, this can be done by careful planning to assure both leads, extensions, and IPGs are positioned into the same coronal plane. In the case where a single double-channel IPG is implanted unilaterally to stimulate both leads, this can be done much easier by overlapping extension cables.

To assess the feasibility of implementing surgically modified lead trajectories, we implanted a 74-year-old female PD patient operated for STN DBS with a dual channel IPG (Medtronic ACTIVA PC, Medtronic Inc., Minneapolis MN) stimulating both left and right leads. To guide the lead trajectory, we used curved mayo scissors passed posterior and to the left of the incision. The blades of the scissors were then opened to their 
Table 1

Maximum of unilateral and bilateral SAR Reduction Efficiency (SREn,i and SREnb) for each patient and their corresponding optimum coil rotating angles.

\begin{tabular}{|c|c|c|c|c|c|c|}
\hline Patients & Coil angle $\left({ }^{\circ}\right)$ & SRE $E_{\text {Right-lead }}(\%)$ & Coil angle $\left({ }^{\circ}\right)$ & SRE $_{\text {Left-lead }}(\%)$ & Coil angle $\left({ }^{\circ}\right)$ & SRE $_{\text {Bilateral }}(\%)$ \\
\hline ID1 & 124 & 98 & 101 & 69 & 124 & 97 \\
\hline ID2 & 90 & 67 & 39 & 47 & 56 & -18 \\
\hline ID3 & 101 & 95 & 113 & 79 & 113 & 86 \\
\hline ID4 & 107 & 83 & 169 & 72 & 124 & 22 \\
\hline ID5 & 56 & 84 & 62 & 96 & 56 & 94 \\
\hline ID6 & 169 & 70 & 90 & 92 & 68 & -15 \\
\hline ID7 & 34 & 77 & 39 & 89 & 39 & 89 \\
\hline ID8 & 17 & 90 & 62 & 96 & 45 & 49 \\
\hline ID9 & 6 & 94 & 39 & 92 & 28 & 52 \\
\hline ID10 & 62 & 67 & 68 & 81 & 68 & 81 \\
\hline ID11 & 62 & 84 & 51 & 88 & 51 & 88 \\
\hline ID12 & 23 & 78 & 45 & 76 & 39 & 71 \\
\hline ID13 & 79 & 98 & 118 & 97 & 101 & 70 \\
\hline Mean & 71 & 83 & 77 & 83 & 70 & 59 \\
\hline Standard deviation & 45 & 11 & 38 & 14 & 32 & 38 \\
\hline
\end{tabular}

widest to create a pocket for a coiled lead to be inserted (Fig. 6). The lead was looped into 2-3 concentric circles at the burr-hole and the rest of the lead was passed toward the temporal lobe where it would be later connected to the extension. The IPG was implanted in the left pectoralis (Fig. 7). Lead and extension trajectories were aligned and overlapped as shown in Fig. 7. A head and chest CT image of the patient was acquired after the full system was implanted and lead and extension trajectories were extracted from the image for model construction and simulations similar to previous patients.

Fig. 8 gives the maximum of 1 -averaged SAR at the tips of left and right DBS leads as a function of coil's rotation angle. The SAR values generated by the body coil are also given for comparison. As expected, when lead trajectories are surgically overlapped, the SAR profile of left and right leads vary in phase. A maximum bilateral SRE of 95\% could be achieved in this case for the coil positioned at $\varphi=95.625^{\circ}$.

\section{Sensitivity to operational errors}

From Fig. 5 it can be observed that large deviations from coil's optimal angle generate a SAR that is higher than the SAR produced by the body coil (when normalized to the same $\mathrm{B}_{1}^{+}$). This has important safety implications considering both operational errors and uncertainties in determining the optimum angle. To assess the sensitivity of SAR to deviation of the coil from its optimal position we defined a metric called permissible rotation range (PRR) as the maximum deviation from the optimal position in either direction that increased the SAR to the level produced by the $\mathrm{CP}$ coil:

$\mathrm{PRR}_{\mathrm{i}}=\left|\varphi_{\mathrm{i}, \mathrm{opt}}-\varphi_{\mathrm{i}, \mathrm{CP}}\right|$. where $\varphi_{\mathrm{i}, \mathrm{opt}}$ is the optimum angle for lead $\mathrm{i}$ and $\varphi_{\mathrm{i}, \mathrm{CP}}$ is the angle that produces the SAR equal to the SAR generated by the CP coil. PRR was $20.16^{\circ} \pm 12.78^{\circ}$ averaged over leads. For a coil constructed such that it allows rotational increments of $5^{\circ}$ (which is practical) the PRR will be at least 4 times larger than coil's rotation resolution.

\section{Effect of electrical properties of the tissue on optimal coil position}

A possible approach to determine the optimal position of the coil for individual patients is to perform simulations on patient-derived models of implanted device to determine the coil position that minimizes the SAR for each individual. If such simulation approach is adopted, it will be important to evaluate the sensitivity of coil's positioning to electrical properties of the body model. To assess this, we repeated simulations in two cases with (a) a patient with isolated bilateral leads (ID5, Fig. 3) and (b) the patient with fully-implanted system and a double-channel unilateral IPG (ID14, Fig. 6), with a range of low to high tissue conductivities covering the limits reported in the literature $(0.2 \mathrm{~S} / \mathrm{m}-0.65 \mathrm{~S} / \mathrm{m})$. Fig. S2 of supplemental material gives the result of Max1gSAR as a function of coil rotating angle for each tissue conductivity. As expected, the absolute value of the SAR changed by varying the conductivity of the tissue. However, the position of coil's optimum angle was insensitive to the electrical properties of body model $\left(<1^{\circ}\right.$ change).

\section{Temperature rise in the tissue during typical clinical scans}

Although local SAR has been widely used as a surrogate to predict the severity of RF heating during MRI, temperature rise in the tissue $(\Delta \mathrm{T})$ is

(A)
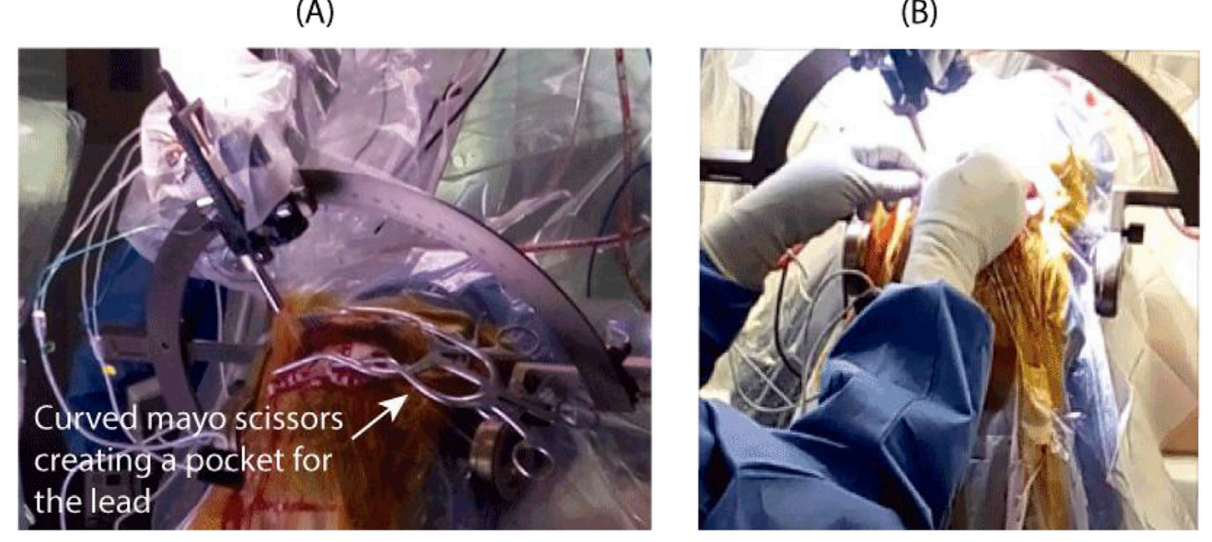

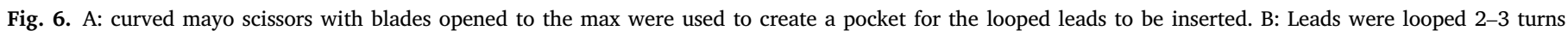
concentrically and placed on top of the surgical burr hole. A video demonstrating forming and positioning of the loop is given in the supplementary material. 


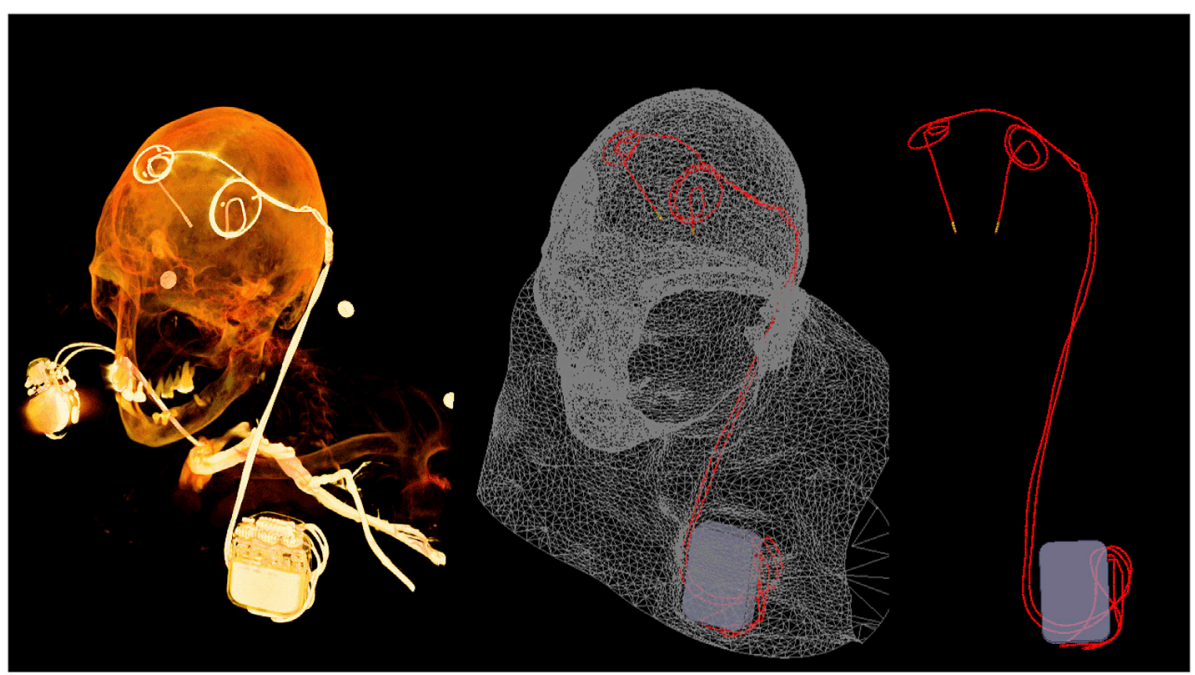

Fig. 7. Postoperative CT image of a patient implanted with bilateral DBS leads connected to a doublechannel pulse generator implanted in the right pectoral region. Concentric loops were incorporated into lead trajectories at the surgical burr hole following the technique described in (Golestaniradet al, 2019a). The rest of the lead trajectories and extension cables were aligned and overlapped to follow the same path. A model of the patient's silhouette and the fully implanted device was constructed based on the CT image for finite element simulations.

ID14

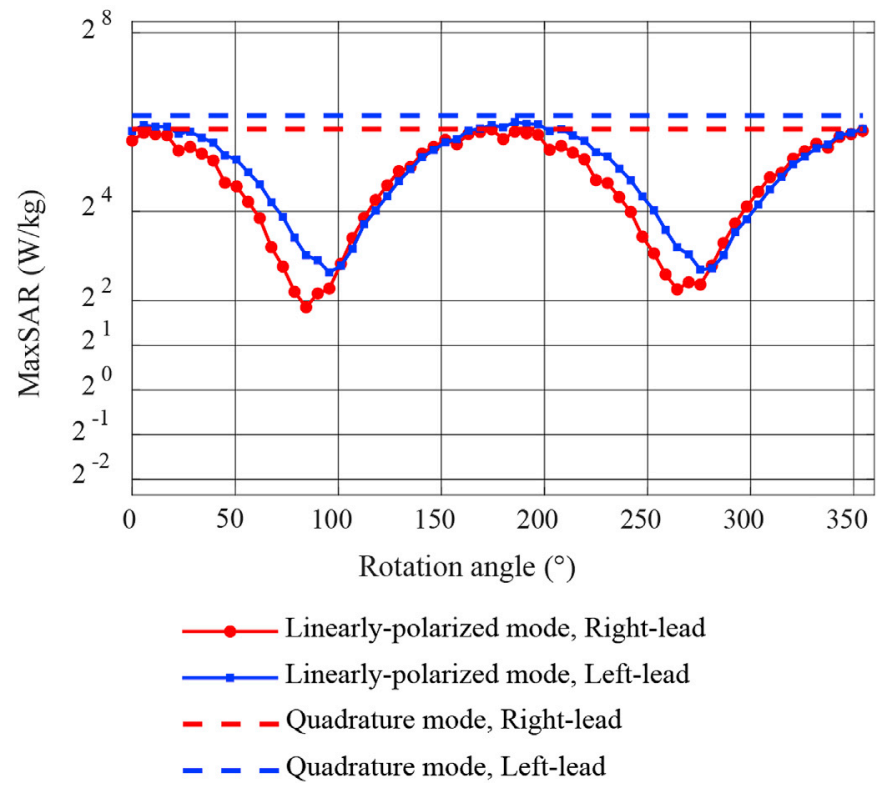

Fig. 8. The maximum of 1 -averaged SAR calculated around tips of left and right DBS leads of patient \#14 as a function of rotating coil angle $\varphi^{\circ}$ (solid lines). The input power of the coil is adjusted to generate a mean $\mathrm{B}_{1}^{+}=2 \mu \mathrm{T}$ on a central axial plane. The maximum of 1g-averaged SAR is also given for the body coil generating the same $\mathrm{B1}^{+}$(dashed-lines). As expected, when lead trajectories are overlapped the SAR profiles of right and left leads vary very similarly as a function of coil rotation angle. This significantly enhances bilateral SAR reduction by allowing simultaneous SAR minimization at tips of both leads at a common optimum angle.

the ultimate measure of RF safety that dictates the limits. To have an estimate of rotating coil's performance in terms of reducing the temperature rise in tissue we calculated $\Delta \mathrm{T}$ around tips of left and right leads in patient 14 during eight typical MRI exams. Simulations were performed for the rotating coil positioned at its optimum angle as well as the scanner's built-in body coil, where the input power to both coils was adjusted to produce the same flip angle on a central axial plane passing through the head. Table 2 gives the sequences and their parameters. Combined electromagnetic-thermal simulation were performed as described in our previous work (Golestaniradet al, 2019c). Scan parameters of Table 2 are based on typical clinical sequences on a Siemens $3 \mathrm{~T}$
Skyra system that we routinely use in our center. Thermal simulations were performed using the transient thermal analyses solver of ANSYS Mechanical (ANSYS Inc., Canonsburg, PA) which solves the Penne bio heat equation:

$\operatorname{co} \frac{\partial \mathrm{T}}{\partial \mathrm{t}}-\nabla \mathrm{k} \nabla \mathrm{T}=\rho(\mathrm{SAR})$

where $\mathrm{c}$ is the specific heat capacity of the tissue (4150 Jkg-1C- $1 \mathrm{~W} / \mathrm{m} / \mathrm{C}$ (Cheng and Plewes, 2002)), $\mathrm{k}$ is the isotropic thermal conductivity (0.42 W/m-1C-1 (Cheng and Plewes, 2002)), $\rho$ is the density $(1000 \mathrm{~kg} / \mathrm{m} 3)$, and $\mathrm{T}$ is the temperature. The initial body temperature was set to $37^{\circ} \mathrm{C} . \Delta \mathrm{T}$ in the tissue generated by the rotating coil in its optimum angle remained below $1^{\circ} \mathrm{C}$ for all imaging sequences whereas temperature increases as high as $10.8^{\circ} \mathrm{C}$ was reached during imaging with the body coil.

\section{Discussion and conclusion}

A significant obstacle to develop and test hypotheses on the mechanism of action of DBS is the lack of a quantitative understanding of the extent and influence of the stimulation on the neural elements involved. Much of the scientific effort to address this question has focused on the cellular effects of stimulation near the electrode (Butson and McIntyre, 2006; Chaturvedi et al., 2010; Grill, 2001; McIntyre et al., 2004a; McIntyre et al., 2004b; Miocinovicet al, 2006; Golestanirad et al., 2013; Golestanirad et al., 2012; Golestaniradet al, 2018). While this interesting question continues to be explored, it is proposed that it may not be the fundamental issue underlying the therapeutic mechanisms of DBS, and that changes in the underlying dynamics of the stimulated brain networks may represent the intervention's core effect (McIntyre and Hahn, 2010). When investigating the neuromodulatory effects of DBS, neuroimaging studies have largely used either positron emission tomography (PET) or single photon emission tomography (Geday et al., 2009; Ciliaet al, 2009). MRI has clear advantages to both of these techniques due to its excellent soft-tissue contrast, easy access, non-invasive nature, and the richness of the post-processing analytical methods that are available to use. Present MRI technology however, is limited in its post-operative applicability for DBS patients due to safety risks.

In the past few years, manufacturers of electronic implantable medical devices have largely reduced the use of ferromagnetic components to diminish the risk of device dislodgement due to static magnetic fields. Device programming has been also significantly enhanced to minimize the risk of malfunction due to interference from gradient fields. RF heating however, remains a major challenge. Although some 
Table 2

Sequences and scan parameters used in typical brain exams and in thermal simulations. Here TR refers to repetition time, TE to echo time, WE to bandwidth, FOV to field of view, FA to flip angle, and ST to slice thickness.

\begin{tabular}{|c|c|c|c|c|c|c|c|}
\hline Index & Sequence & $\begin{array}{l}\text { TR } \\
(\mathrm{ms})\end{array}$ & $\begin{array}{l}\mathrm{TE} \\
(\mathrm{ms})\end{array}$ & $\begin{array}{l}\text { FOV } \\
(\mathrm{cm})\end{array}$ & $\begin{array}{l}\text { B1+ } \\
\text { (uT) }\end{array}$ & $\begin{array}{l}\text { Acq. } \\
\text { Time }\end{array}$ & $\begin{array}{l}\Delta \mathrm{T}\left[{ }^{\circ} \mathrm{C}\right] \\
\text { Body coil/ } \\
\text { LP coil }\end{array}$ \\
\hline 1 & Localizer & 8.6 & 4 & 250 & 0.4 & $0: 13$ & $\begin{array}{l}<0.1^{\circ} / \\
<0.1^{\circ}\end{array}$ \\
\hline 2 & $\begin{array}{l}\text { AX T1 } \\
\text { TSE }\end{array}$ & 486 & 13 & 200 & 2.4 & $2: 46$ & $6.9^{\circ} / 0.3^{\circ}$ \\
\hline 3 & $\begin{array}{l}\text { AX T2 } \\
\text { TSE }\end{array}$ & 4500 & 83 & 240 & 2.8 & $3: 47$ & $10.8^{\circ} / 0.7^{\circ}$ \\
\hline 4 & AX FLAIR & 9000 & 81 & 220 & 1.7 & $2: 44$ & $2.2^{\circ} / 0.2^{\circ}$ \\
\hline 5 & AX CISS & 8.21 & 3.81 & 180 & 2.4 & $5: 30$ & $8.0^{\circ} / 0.4^{\circ}$ \\
\hline 6 & $\begin{array}{l}\text { AX 3D } \\
\text { TOF }\end{array}$ & 21 & 3.43 & 200 & 2.3 & $5: 52$ & $7.1^{\circ} / 0.5^{\circ}$ \\
\hline 7 & Diffusion & 6600 & 99 & 220 & 1.3 & $1: 54$ & $2.0^{\circ} / 0.1^{\circ}$ \\
\hline 8 & fMRI & 2020 & 20 & 170 & 0.9 & $8: 33$ & $1.3^{\circ} /<0.1^{\circ}$ \\
\hline
\end{tabular}

manufacturers have relaxed their guidelines to allow the use of body coils which was previously prohibited, major practical challenges are virtually unaffected as stringent power monitoring remains in place (new guidelines limit the maximum rms of $\mathrm{B}_{1}^{+}$field to $2 \mu \mathrm{T}$ and in cases where the scanner does not report the B field, the more conservative whole-head SAR limit of $0.1 \mathrm{~W} / \mathrm{kg}$ should be applied). Considerable effort has been dedicated to understand and control safety risks by characterizing the problem of RF heating accounting for factors such as lead configuration (Rezaiet al, 2004; Baker et al., 2005; Golestanirad et al., 2016; Shrivastavaet al, 2010), position with respect to MRI RF coil (Nitz et al., 2001; Matteiet al, 2008; Golestaniradet al, 2019c) and lead material (Golestaniradet al, 2019b; Serano et al., 2015). There is however, a consensus that MRI RF safety is a complex phenomenon with a plethora of interplaying factors. As such, it should be tackled from different fronts, meaning that efforts in designing MR-compatible leads, novel DBS-friendly MRI coils, and surgical lead management should be combined together to mitigate the risks effectively.

Recently we introduced the concept of reconfigurable MRI coil technology for low-SAR imaging of patients with DBS implants at $1.5 \mathrm{~T}$. In this work we demonstrated the feasibility of advancing the technology to 3T where MRI can render major benefits, and for the first time assessed the possibility of including patients with bilateral leads and fully implanted systems which are most common practices.

\subsection{Inter-subject variability of implanted lead trajectories and the role of surgical planning}

During the past 5 years we have examined postoperative CT images of more than 120 patients with DBS leads for numerical simulations assessing the SAR. These patients have been operated at three different centers in Massachusetts General Hospital, Albany Medical Center and Northwestern Memorial Hospital, and by at least four different neurosurgeons. A striking observation was the substantial patient-to-patient variation in the trajectory of extra cranial portion of the leads (see Fig. 3). Unlike the meticulously planned intracranial trajectory of the electrode, for which almost every neurosurgeon follows textbook guidelines to determine the entry point on the skull and chose the angle of penetration to the target nuclei, there are no guidelines for the placement of extra cranial portions of the leads. This causes a large variation in lead trajectories depending on surgeons' preference and practice style. In the context of rotating coil technology, such variation poses a problem for simultaneous reduction of the SAR at tips of bilateral leads as it can be observed from Fig. 5. In cases where left and right leads have non-overlapping trajectories, the optimum rotation angle that minimizes the SAR for one lead could be far from optimum for the other lead. A relatively simple surgical modification can address this problem by aligning and overlapping trajectories of left and right leads and extension cables. This work demonstrated the first results of such modification implemented in a patient who was implanted with a bilateral lead system. The bilateral SAR Reduction Efficiency (SRE) of the coil was substantially higher for the patient with modified lead trajectories compared to the average in patients who were operated without any specific instruction (95\% vs 59\%). An interesting observation was that the SAR generated by the rotating coil at all rotation angles was always lower than the SAR generated by the body coil in the patient with modified DBS trajectory. This is a significant result as it suggests surgical modifications can significantly reduce the risks associates with operational errors when the rotating coil is used on patients. In general, rotating head coil significantly outperformed the body coil in patients with fully-implanted systems (ID13 and ID14) which was predictable considering a substantial portion of the DBS device will be outside of head coil's field of view.

\subsection{Limitations of DBS MRI in clinical settings}

Currently Abbott (Abbott Laboratories, Chicago IL) is the only manufacturer of MR-conditional directional DBS leads that allow for current steering and selective target stimulation for which postoperative fMRI will provide invaluable information on the mechanism of action of stimulation. MRI labeling of Abbott DBS systems limits the $\mathrm{B}_{1}^{+}$to $1.3 \mu \mathrm{T}$ for fully implanted systems. From Table 2 it can be observed that this value is well exceeded in many typical MRI brain exams. For example, running a T2-weighted turbo spin echo sequence which is the gold standard protocol for stroke diagnosis produced $10.8^{\circ}$ temperature rise when the body coil was used, whereas the rotating head coil generated $<1^{\circ}$ heating. Other useful but high-SAR sequences are time of flight angiography and constructive interference in steady state (CISS) MRI. CISS MRI in particular, is widely used in evaluation of the cranial nerves, CSF rhinorrhea and aqueduct stenosis (Hingwala et al., 2011). Both sequences generated $>7^{\circ}$ temperature rise in simulations with the body coil and $<0.5^{\circ}$ heating when the rotating coil was used in its optimum angle. It is important to note that although our simulations were performed using simplified DBS lead models which does not reflect the actual temperature rise of the commercial device, they still give a fair comparative view of RF heating generated by MRI body coil and the rotating coil system.

This work presents a feasibility study to scale the reconfigurable MRI coil technology for low SAR imaging of DBS patients at 3T. We performed finite element simulations using patient-derived models of DBS systems with realistic device configurations. Both isolated leads and fully implanted systems were evaluated. A total of 832 simulations were performed to evaluate the SAR reduction performance of a rotating 3T head coil currently under construction in our lab. Our results showed a promising SAR reduction efficiency of $>80 \%$ for unilateral leads and $>59 \%$ for bilateral leads. A simple surgical modification in implantation of DBS leads boosted the coil's SAR reduction efficiency to $>90 \%$, leading to a 15-fold reduction in temperature rise in the tissue during typical MRI exams compared to a CP body coil. Our results suggest that the reconfigurable MRI technology offers a promising solution to the problem of RF safety of deep brain stimulation implants at $3 \mathrm{~T}$.

\section{Disclaimer}

The mention of commercial products, their sources, or their use in connection with material reported herein is not to be construed as either an actual or implied endorsement of such products by the Department of Health and Human Services.

\section{Acknowledgment}

This work was supported by the NIH grant R00EB021320. 
Appendix A. Supplementary data

Supplementary data to this article can be found online at https://doi. org/10.1016/j.neuroimage.2019.05.015.

\section{References}

Baker, K.B., Tkach, J., Hall, J.D., Nyenhuis, J.A., Shellock, F.G., Rezai, A.R., 2005. Reduction of magnetic resonance imaging-related heating in deep brain stimulation leads using a lead management device. Neurosurgery 57 (4), 392-397.

Blomstedt, P., Hariz, G.-M., Hariz, M.I., Koskinen, L.-O., 2007. Thalamic deep brain stimulation in the treatment of essential tremor: a long-term follow-up. Br. J. Neurosurg. 21 (5), 504-509.

Boccard, S.G., Pereira, E.A., Moir, L., Aziz, T.Z., Green, A.L., 2012. Long-term outcomes of deep brain stimulation for neuropathic pain. Neurosurgery 72 (2), 221-231.

Boon, P., et al., 2007. Deep brain stimulation in patients with refractory temporal lobe epilepsy, 48 (8), 1551-1560.

Buchli, R., Boesiger, P., Meier, D., 1988. Heating effects of metallic implants by MRI examinations. Magn. Reson. Med. 7 (3), 255-261.

Butson, C.R., McIntyre, C.C., 2006. Role of electrode design on the volume of tissue activated during deep brain stimulation. J. Neural Eng. 3 (1), 1-8.

Chaturvedi, A., Butson, C.R., Lempka, S.F., Cooper, S.E., McIntyre, C.C., 2010. Patientspecific models of deep brain stimulation: influence of field model complexity on neural activation predictions. Brain Stimul. 3 (2), 65-77.

Cheng, H.L.M., Plewes, D.B., 2002. Tissue thermal conductivity by magnetic resonance thermometry and focused ultrasound heating. J. Magn. Reson. Imaging 16 (5), 598-609.

Chou, C.-K., McDougall, J.A., Chan, K.W., 1997. RF heating of implanted spinal fusion stimulator during magnetic resonance imaging. IEEE Trans. Biomed. Eng. 44 (5), 367-373.

Cilia, R., et al., 2009. Clinical and cerebral activity changes induced by subthalamic nucleus stimulation in advanced Parkinson's disease: a prospective case-control study. Clin. Neurol. Neurosurg. 111 (2), 140-146.

Cui, Z., Ling, Z., 2016. A paradigm shift toward MRI-guided and MRI-verified DBS surgery Response. J. Neurosurg. 124 (4), 1137-1138.

Eryaman, Y., Akin, B., Atalar, E., 2011. Reduction of implant RF heating through modification of transmit coil electric field. Magn. Reson. Med. 65 (5), 1305-1313.

Eryaman, Y., et al., 2014. Parallel transmit pulse design for patients with deep brain stimulation implants. Magn. Reson. Med. 73 (5), 1896-1903.

Eryaman, Y., et al., 2019. A simple geometric analysis method for measuring and mitigating RF induced currents on Deep Brain Stimulation leads by multichannel transmission/reception. Neuroimage 184, 658-668.

Feinberg, D.A., Setsompop, K., 2013. Ultra-fast MRI of the human brain with simultaneous multi-slice imaging. J. Magn. Reson. 229, 90-100.

Flora, E.D., Perera, C.L., Cameron, A.L., Maddern, G.J., 2010. Deep brain stimulation for essential tremor: a systematic review. Mov. Disord. 25 (11), 1550-1559.

Gabriels, L., Cosyns, P., Nuttin, B., Demeulemeester, H., Gybels, J., 2003. Deep brain stimulation for treatment-refractory obsessive compulsive disorder: phsychopathological and neuropsychological outcome in three cases. Acta Psychiatr. Scand. 107, 275-282.

Geday, J., Østergaard, K., Johnsen, E., Gjedde, A., 2009. STN-stimulation in Parkinson's disease restores striatal inhibition of thalamocortical projection. Hum. Brain Mapp. 30 (1), 112-121.

Golestanirad, L., Izquierdo, A.P., Graham, S.J., Mosig, J.R., Pollo, C., 2012. Effect of realistic modeling of deep brain stimulation on the prediction of volume of activated tissue. Progr. Electromag. Res. 126, 1-16.

Golestanirad, L., Elahi, B., Molina Arribere, A., Mosig, J.R., Pollo, C., Graham, S.J., 2013 Analysis of fractal electrodes for efficient neural stimulation. Front. Neuroeng. 6 (3).

Golestanirad, L., Angelone, L.M., Iacono, M.I., Katnani, H., Wald, L.L., Bonmassar, G. 2016. Local SAR near deep brain stimulation (DBS) electrodes at $64 \mathrm{MHz}$ and 127 MHz: a simulation study of the effect of extracranial loops. Magn. Reson. Med. 88 (4), 1558-1565.

Golestanirad, L., Keil, B., Angelone, L.M., Bonmassar, G., Mareyam, A., Wald, L.L., 2017. Feasibility of using linearly polarized rotating birdcage transmitters and close-fitting receive arrays in MRI to reduce SAR in the vicinity of deep brain simulation implants. Magn. Reson. Med. 77 (4), 1701-1712.

Golestanirad, L., et al., 2016. A Patient-adjustable MRI coil for implant-friendly imaging of deep brain stimulation: design, construction, and patient-specific numerical simulations. Proc. Intl. Soc. Mag. Reson. Med. 24.

Golestanirad, L., et al., 2017. Construction and modeling of a reconfigurable MRI coil for lowering SAR in patients with deep brain stimulation implants. Neuroimage 147, 577-588.

Golestanirad, L., et al., 2019. Changes in the specific absorption rate (SAR) of radiofrequency energy in patients with retained cardiac leads during MRI at $1.5 \mathrm{~T}$ and 3T. Magn. Reson. Med. 81, 653-669.

Golestanirad, L., et al., 2018. Solenoidal micromagnetic stimulation enables activation of axons with specific orientation. Front. Physiol. 9.

Golestanirad, L., et al., 2019. RF-induced heating in tissue near bilateral DBS implants during MRI at 1.5 T and 3T: the role of surgical lead management. Neuroimage 184, $566-576$.

Golestanirad, L., et al., 2019. Reducing RF-induced heating near implanted leads through high-dielectric capacitive bleeding of current (CBLOC). IEEE Trans. Microw. Theory Tech. 67, 1265-1273.
Greenberg, B., et al., 2008. Deep brain stimulation of the ventral internal capsule/ventral striatum for obsessive-compulsive disorder: worldwide experience. Mol. Psychiatry 15 (1), 64-79.

Grill, W., 2001. Extracellular excitation of central neurons: implications for the mechanisms of deep brain stimulation. Thalamus Relat. Syst. 1 (3), 269-277.

Hancu, I., et al., 2018. On the (Non-) Equivalency of Monopolar and Bipolar Settings for Deep Brain Stimulation fMRI Studies of Parkinson's Disease Patients.

Hingwala, D., Chatterjee, S., Kesavadas, C., Thomas, B., Kapilamoorthy, T.R., 2011. Applications of 3D CISS sequence for problem solving in neuroimaging. Indian J. Radiol. Imaging 21, 90.

Hubble, J., Busenbark, K., Wilkinson, S., Penn, R., Lyons, K., Koller, W., 1996. Deep brain stimulation for essential tremor. Neurology 46 (4), 1150-1153.

IEEE, 2014. P1528.4TM/D1.0,Recommended Practice for Determining the Peak Spatial Average Specific Absorption Rate (SAR) in the Human Body from Wireless Communications Devices, $30 \mathrm{MHz}$ - 6 GHz: Requirements for Using the FiniteElement Method for SAR Calculations, Specifically Involving Vehicle Mounted Antennas and Personal Wireless Devices.

Jude Medical, St, 2018. MRI Procedure Information. https://manuals.sjm.com.

Kerl, H.U., Gerigk, L., Pechlivanis, I., Al-Zghloul, M., Groden, C., Nölte, I., 2012. The subthalamic nucleus at 3.0 Tesla: choice of optimal sequence and orientation for deep brain stimulation using a standard installation protocol. J. Neurosurg. 117 (6), 1155-1165.

Kozlov, M., Turner, R., 2009. Fast MRI coil analysis based on 3-D electromagnetic and RF circuit co-simulation. J. Magn. Reson. 200 (1), 147-152.

Kumar, R., Dagher, A., Hutchison, W., Lang, A., Lozano, A., 1999. Globus pallidus deep brain stimulation for generalized dystonia: clinical and PET investigation. Neurology 53 (4), 871-871.

Laxton, A.W., Lozano, A. M. J. W. n., 2013. Deep brain stimulation for the treatment of Alzheimer disease and dementias, 80 (3-4), S28. e1-S28. e8.

Lozano, A.M., et al., 2019. Deep brain stimulation: current challenges and future directions. Nat. Rev. Neurol. 1.

Mattei, E., et al., 2007. Temperature and SAR measurement errors in the evaluation of metallic linear structures heating during MRI using fluoroptic ${ }^{\circledR}$ probes. Phys. Med. Biol. 52 (6), 1633.

Mattei, E., et al., 2008. Complexity of MRI induced heating on metallic leads: experimental measurements of 374 configurations. Biomed. Eng. Online 7 (1), 11.

McElcheran, Clare, Golestanirad, L., Graham, S., 2014. Reduced heating of implanted electrical conductors using parallel radiofrequency transmission. In: Joint Annual Meeting of the International Society of Magnetic Resonace in Medicin. ISMRM), Milan, Italy.

McElcheran, C.E., Yang, B., Anderson, K.J., Golenstani-Rad, L., Graham, S.J., 2015. Investigation of parallel radiofrequency transmission for the reduction of heating in long conductive leads in 3 tesla magnetic resonance imaging. PLoS One 10 (8), e0134379.

McElcheran, C., Golestani-Rad, L., Graham, S., 2016. Heating reduction in unilateral and bilateral implanted leads at 3T using parallel radiofrequency transmission in a heterogeneous head model. Proc. Intl. Soc. Mag. Reson. Med. 24.

McElcheran, C.E., Yang, B., Anderson, K.J., Golestanirad, L., Graham, S.J., 2017. Parallel radiofrequency transmission at 3 tesla to improve safety in bilateral implanted wires in a heterogeneous model. Magn. Reson. Med. 78 (6), 2406-2415.

McElcheran, C.E., Yang, B., Anderson, K.J., Golestanirad, L., Graham, S.J., 2017. Parallel radiofrequency transmission at 3 tesla to improve safety in bilateral implanted wires in a heterogeneous model. J. Magn. Reson. Med. 78 (6), 2406-2415.

McElcheran, C., et al., 2017. Parallel transmission for heating reduction in realistic deep brain stimulation lead trajectories. Proc. Intl. Soc. Mag. Reson. Med. 25.

McElcheran, C., et al., 2019. Numerical simulations of realistic lead trajectories and an experimental verification support the efficacy of parallel radiofrequency transmission to reduce heating of deep brain stimulation implants during MRI. Nat. Sci. Rep. 9 (1), 2124.

McIntyre, C.C., Hahn, P.J., 2010. Network perspectives on the mechanisms of deep brain stimulation. Neurobiol. Dis. 38 (3), 329-337.

McIntyre, C.C., Grill, W.M., Sherman, D.L., Thakor, N.V., 2004. Cellular effects of deep brain stimulation: model-based analysis of activation and inhibition. J. Neurophysiol. 91 (4), 1457-1469.

McIntyre, C.C., Mori, S., Sherman, D.L., Thakor, N.V., Vitek, J.L., 2004. Electric field and stimulating influence generated by deep brain stimulation of the subthalamic nucleus. Clin. Neurophysiol. 115 (3), 589-595.

Medtronic, 2015. MRI Guidelines for Medtronic Deep Brain Stimulation Systems. http://manuals.medtronic.com/wcm/groups/mdtcom_sg/@emanuals/@era/@neu ro/documents/documents/contrib_228155.pdf.

Miocinovic, S., et al., 2006. Computational analysis of subthalamic nucleus and lenticular fasciculus activation during therapeutic deep brain stimulation. J. Neurophysiol. 96 (3), 1569-1580.

Nitz, W.R., Oppelt, A., Renz, W., Manke, C., Lenhart, M., Link, J., 2001. On the heating of linear conductive structures as guide wires and catheters in interventional MRI. J. Magn. Reson. Imaging 13 (1), 105-114.

Nordbeck, P., et al., 2009. Measuring RF-induced currents inside implants: impact of device configuration on MRI safety of cardiac pacemaker leads. Magn. Reson. Med. 61 (3), 570-578.

Ondo, W., Almaguer, M., Jankovic, J., Simpson, R.K., 2001. Thalamic deep brain stimulation: comparison between unilateral and bilateral placement. Arch. Neurol. 58 (2), 218-222.

Ostrem, J.L., Starr, P.A., 2008. Treatment of dystonia with deep brain stimulation. Neurotherapeutics 5 (2), 320-330.

Owen, S., Green, A., Nandi, D., Bittar, R., Wang, S., Aziz, T.Z., 2007. Deep brain stimulation for neuropathic pain. In: Operative Neuromodulation. Springer, pp. 111-116. 
Park, S.M., Kamondetdacha, R., Nyenhuis, J.A., 2007. Calculation of MRI-induced heating of an implanted medical lead wire with an electric field transfer function. J. Magn. Reson. Imaging 26 (5), 1278-1285.

Ramani, S., Schulte, R., Mckinnon, G., Ashe, J., Pilitsis, J., Hancu, I., 2018. Accurate localization of individual DBS contacts by MRI using zero-TE phase images. Proc. Intl. Soc. Mag. Reson. Med. 26.

Rezai, A.R., et al., 2004. Neurostimulation system used for deep brain stimulation (DBS): MR safety issues and implications of failing to follow safety recommendations. Investig. Radiol. 39 (5), 300-303.

Rezai, A.R., et al., 2005. Is magnetic resonance imaging safe for patients with neurostimulation systems used for deep brain stimulation? Neurosurgery 57 (5), 1056-1062.

Serano, P., Angelone, L.M., Katnani, H., Eskandar, E., Bonmassar, G., 2015. A novel brain stimulation technology provides compatibility with MRI. Sci. Rep. 5.

Shrivastava, D., et al., 2010. Effect of the extracranial deep brain stimulation lead on radiofrequency heating at 9.4 Tesla (400.2 MHz). J. Magn. Reson. Imaging 32 (3), 600-607.
Taba, H.A., et al., 2010. A closer look at unilateral versus bilateral deep brain stimulation: results of the National Institutes of Health COMPARE cohort. J. Neurosurg. 113 (6), 1224-1229.

Wei, P., Yang, B., McElcheran, C., Golestanirad, L., Graham, S., 2018. Reducing radiofrequency-induced heating in realistic deep brain stimulation lead trajectories using parallel transmission. Proc. Intl. Soc. Mag. Reson. Med. 26.

Williams, N.R., Taylor, J.J., Lamb, K., Hanlon, C.A., Short, E.B., George, M.S., 2014. Role of functional imaging in the development and refinement of invasive neuromodulation for psychiatric disorders. World J. Radiol. 6 (10), 756.

Yeo, D.T., Wang, Z., Loew, W., Vogel, M.W., Hancu, I., 2011. Local SAR in high pass birdcage and TEM body coils for multiple human body models in clinical landmark positions at 3T. J. Magn. Reson. Imaging: JMRI 33 (5), 1209.

Yeung, C.J., Susil, R.C., Atalar, E., 2002. RF heating due to conductive wires during MRI depends on the phase distribution of the transmit field. Magn. Reson. Med. 48 (6), 1096-1098. 